\title{
Multi-Hamiltonian Structures on Beauville's Integrable System and Its Variant ${ }^{\star}$
}

\author{
Rei INOUE ${ }^{\dagger}$ and Yukiko KONISHI $\ddagger$ \\ $\dagger$ Department of Physics, Graduate School of Science, The University of Tokyo, \\ 7-3-1 Hongo, Bunkyo, Tokyo 113-0033, Japan \\ E-mail: reiiy@monet.phys.s.u-tokyo.ac.jp \\ $\ddagger$ Graduate School of Mathematical Sciences, The University of Tokyo, \\ 3-8-1 Komaba, Meguro, Tokyo 153-8914, Japan \\ E-mail: konishi@ms.u-tokyo.ac.jp
}

Received October 24, 2006, in final form December 29, 2006; Published online January 08, 2007 Original article is available at http://www.emis.de/journals/SIGMA/2007/007/

\begin{abstract}
We study Beauville's completely integrable system and its variant from a viewpoint of multi-Hamiltonian structures. We also relate our result to the previously known Poisson structures on the Mumford system and the even Mumford system.
\end{abstract}

Key words: completely integrable system; Mumford system; multi-Hamiltonian structure

2000 Mathematics Subject Classification: 37J35; 14H70

\section{Introduction}

Let $r \geq 2$ and $d \geq 1$ be integers. In [1], Beauville constructed an algebraically completely integrable Hamiltonian system on the space of the gauge equivalence classes of $r \times r$ polynomial matrices whose degree is less than or equal to $d$. This system is a generalization of the Mumford system [6], and the general level set is isomorphic to the complement of the theta divisor in the Jacobian variety of the spectral curve. Employing Beauville's approach, a variant is constructed in [5], which gives a generalization of the even Mumford system introduced by Vanhaecke [11, 12]. The remarkable difference with Beauville's system is that the general level set is isomorphic to the complement of the intersection of $r$ translates of the theta divisor. We call these systems the Beauville system and the Beauville-Vanhaecke (BV) system in this paper.

The Poisson structures of these systems depend on a monic polynomial of degree $d+2$ having only simple roots. Our first aim in this article is to understand them in terms of the multiHamiltonian structure (see $[9, \S 4$ and $\S 12]$ ). This is achieved by extending them to those parameterized by a polynomial $\phi(x)$ of degree at most $d+2 ; \phi(x)$ need not be monic nor have simple roots, and $\operatorname{deg} \phi(x)$ can be less than $d+2$ (Propositions 1 and 3 ). It then turns out that the Poisson structures corresponding to $\phi(x)=1, x, \ldots, x^{d+2}$ give the integrable multi-Hamiltonian system (Theorems 1 and 2).

Since the Beauville system and the BV system are given by the quotient construction, it is an important problem to construct their spaces of representatives and to describe the vector fields and the Poisson structures on them. For the Beauville system, a space of representatives was constructed by Donagi and Markman for a certain subspace [2]. The vector fields on it was computed by $\mathrm{Fu}[4]$ and the multi-Hamiltonian structure was constructed in [8] using the group-theoretic approach. For the BV system, spaces of representatives were given in [5] and the vector fields for $r=2$ and 3 were also given.

*This paper is a contribution to the Vadim Kuznetsov Memorial Issue "Integrable Systems and Related Topics". The full collection is available at http://www.emis.de/journals/SIGMA/kuznetsov.html 
Our second aim is to study the family of Poisson structures on the space of representatives. For the Beauville system, we introduce a new space of representatives for a certain subspace of codimension one which includes Donagi-Markman's result (Proposition 5). In the simplest case of $r=2$ we write down the Poisson structures on this space and on the spaces of representatives for the BV system (Propositions 2 and 4) and compare them with previously known Poisson structures on the Mumford system and the even Mumford system [3, 7, 8].

This article is organized as follows. In Sections 2 and 3, we study Poisson structures and multiHamiltonian structures for the Beauville system and the BV system. We also give expressions of the Poisson structures on the spaces of representatives in the case $r=2$ and compare them with those of the Mumford system and the even Mumford system. Section 4 is devoted to the construction of the new space of the representatives for the subset of the Beauville system.

\section{Multi-Hamiltonian structure on the Beauville system}

We fix numbers $r \in \mathbb{Z}_{\geq 2}$ and $d \in \mathbb{Z}_{\geq 1}$. Throughout this paper, we use the following notations: $S_{d} \subset \mathbb{C}[x]$ denotes the set of polynomials of degree at most $d . E_{i j} \in M_{r}(\mathbb{C})$ is the matrix whose $(i, j)$-th entry is one and other entries are zero. For a matrix $A(x) \in M_{r}(\mathbb{C}[x])$ with polynomial entries,

$$
\begin{array}{ll}
A_{i j}(x) \in \mathbb{C}[x]: & \text { the }(i, j) \text {-th entry of } A(x), \\
A_{i j ; k} \in \mathbb{C}: & \text { the coefficient of } x^{k} \text { in } A_{i j}(x), \\
A_{k} \in M_{r}(\mathbb{C}): & \text { the coefficient of } x^{k} \text { in } A(x) .
\end{array}
$$

Let $W$ be a nonsingular algebraic variety.

Definition 1. A Poisson algebra structure on a sheaf of rings $\mathcal{F}$ on $W$ is a morphism $\{\cdot, \cdot\}$ : $\mathcal{F} \times \mathcal{F} \rightarrow \mathcal{F}$ satisfying skew-symmetry, the Leibniz rule, and the Jacobi identity. A Poisson structure on $W$ is a Poisson algebra structure on the structure sheaf $\mathcal{O}_{W}$.

\subsection{The Beauville system}

Let $V(r, d)$ be the set

$$
V(r, d)=\left\{P(x, y)=y^{r}+s_{1}(x) y^{r-1}+\cdots+s_{r}(x) \in \mathbb{C}[x, y] \mid s_{i}(x) \in S_{d i}\right\} .
$$

For $P(x, y) \in V(r, d)$, let $C_{P}$ be the spectral curve obtained by taking the closure of the affine curve $P(x, y)=0$ in the Hirzebruch surface $\mathbb{F}_{d}=\mathbb{P}\left(\mathcal{O}_{\mathbb{P}^{1}} \oplus \mathcal{O}_{\mathbb{P}^{1}}(d)\right)$ of degree $d$. Consider the set $M_{r}\left(S_{d}\right)$ of $r \times r$ matrices with entries in $S_{d}$ and let $\psi$ be the map:

$$
\psi: M_{r}\left(S_{d}\right) \rightarrow V(r, d), \quad A(x) \mapsto \operatorname{det}\left(y \mathbb{I}_{r}-A(x)\right) .
$$

The group $P G L_{r}(\mathbb{C})$ acts on $M_{r}\left(S_{d}\right)$ by conjugation:

$$
P G L_{r}(\mathbb{C}) \ni g: A(x) \mapsto g(A(x))=g^{-1} A(x) g .
$$

Define a subset $M_{r}\left(S_{d}\right)_{i r}$ of $M_{r}\left(S_{d}\right)$ as

$$
M_{r}\left(S_{d}\right)_{i r}=\left\{A(x) \in M_{r}\left(S_{d}\right) \mid C_{\psi(A(x))} \text { is irreducible }\right\} .
$$

Note that the $P G L_{r}(\mathbb{C})$-action is free on $M_{r}\left(S_{d}\right)_{i r}$. Let $\eta: M_{r}\left(S_{d}\right)_{i r} \rightarrow M_{r}\left(S_{d}\right)_{i r} / P G L_{r}(\mathbb{C})$ be the quotient map. The phase space of the Beauville system is $\mathcal{M}(r, d)=M_{r}\left(S_{d}\right)_{i r} / P G L_{r}(\mathbb{C})$. It was shown in [1] that if $P \in V(r, d)$ defines a smooth spectral curve $C_{P}$, then $\psi^{-1}(P) / P G L_{r}(\mathbb{C})$ 
is isomorphic to the complement of the theta divisor in $\operatorname{Pic}^{g-1}\left(C_{P}\right)$, where $g=\frac{1}{2}(r-1)(r d-2)$ is the genus of $C_{P}$.

Define the vector fields $Y_{i}^{(k)}$ on $M_{r}\left(S_{d}\right)$ by

$$
\sum_{i=0}^{d k-1} a^{i} Y_{i}^{(k)}(A(x))=\frac{1}{x-a}\left[A(x), A(a)^{k}\right], \quad \text { for } \quad k=1, \ldots, r-1 .
$$

Here we have identified the tangent space at each $A(x) \in M_{r}\left(S_{d}\right)$ with $M_{r}\left(S_{d}\right)$. In [1], it was shown that $\eta_{*} Y_{i}^{(k)}$ generate the $g$-dimensional space of translation invariant vector fields on $\mathrm{Pic}^{g-1}\left(C_{P}\right)$.

\subsection{Poisson structure}

We use the following shorthand notations:

$$
M^{\bullet}=M_{r}\left(S_{d+1}\right), \quad M=M_{r}\left(S_{d}\right), \quad M_{i r}=M_{r}\left(S_{d}\right)_{i r}, \quad \mathcal{M}=\mathcal{M}(r, d), \quad G=P G L_{r}(\mathbb{C}) .
$$

Extending the result of $[1, \S 5]$, we are to equip $\mathcal{M}$ with a family of compatible Poisson structures depending on a polynomial $\phi(x) \in S_{d+2}$ :

$$
\phi(x)=\sum_{i=0}^{d+2} \sigma_{i} x^{i}
$$

Note that a Poisson structure on $\mathcal{M}$ is equivalent to a Poisson algebra structure on the sheaf of $G$-invariant functions $\mathcal{O}_{M_{i r}}^{G}$ on $M_{i r}$. Moreover, a Poisson algebra structure on $\mathcal{O}_{M}^{G}$ induces that on $\mathcal{O}_{M_{i r}}^{G}$ since $M_{i r}$ is an open subset of $M$.

Consider the following Poisson structure on $M^{\bullet}$ :

$$
\left\{A_{i j}(x), A_{k l}(y)\right\}=\delta_{i, l} \frac{A_{k j}(x) \phi(y)-\phi(x) A_{k j}(y)}{x-y}-\delta_{k, j} \frac{A_{i l}(x) \phi(y)-\phi(x) A_{i l}(y)}{x-y} .
$$

Let $\iota: M \hookrightarrow M^{\bullet}$ be the closed immersion. Let $\mathcal{I}_{M}$ be the ideal sheaf of $\iota$. Writing $\alpha$ for the natural projection $\mathcal{O}_{M} \bullet \rightarrow \mathcal{O}_{M} \bullet / \mathcal{I}_{M}=\iota_{*} \mathcal{O}_{M}$, we set $\mathcal{N}:=\alpha^{-1}\left(\iota_{*} \mathcal{O}_{M}^{G}\right) \subset \mathcal{O}_{M} \bullet$

Proposition 1. $\mathcal{N}$ is a Poisson subalgebra of $\mathcal{O}_{M} \bullet$. This Poisson algebra structure induces that on $\mathcal{N} / \mathcal{N} \cap \mathcal{I}_{M}$, hence on $\mathcal{O}_{M}^{G}$.

Proof. Let $U$ be an open subset of $M^{\bullet}$. Let us write $F, H \in \mathcal{N}(U)$ as

$$
F=f+\sum_{1 \leq i, j \leq r} A_{i j ; d+1} f_{i j}, \quad H=h+\sum_{1 \leq i, j \leq r} A_{i j ; d+1} h_{i j}
$$

with $f, h \in \mathcal{O}_{M} \bullet(U)^{P G L_{r}(\mathbb{C})}$ and $f_{i j}, h_{i j} \in \mathcal{O}_{M} \bullet(U)$. Note that the Hamiltonian vector fields of $A_{i j ; d+1}(1 \leq i, j \leq r)$ are proportional to the vector fields $X_{\left[E_{j i}\right]}(1 \leq i, j \leq r)$ which generate the infinitesimal actions corresponding to $\left[E_{j i}\right] \in \operatorname{Lie} P G L_{r}(\mathbb{C})$ :

$$
\left\{A_{i j ; d+1}, *\right\}=-\sigma_{d+2} X_{\left[E_{j i}\right]} \quad(1 \leq i, j \leq r) .
$$

Note also that $P G L_{r}(\mathbb{C})$-invariant functions $f, h$ vanish when one applies $X_{\left[E_{j i}\right]}$ as derivations. Combining these facts, we obtain

$$
\{F, H\}=\{f, h\}+\sum_{1 \leq i, j \leq r} A_{i j ; d+1} l_{i j}
$$


where $l_{i j} \in \mathcal{O}_{M} \bullet(U)$. Since (2) is $G$-invariant, $\{f, h\} \in \mathcal{O}_{M \bullet}^{G} \bullet$ Thus we have

$$
\{F, H\} \in \mathcal{N}(U) \text {, and } \alpha(\{F, H\})=\alpha(\{f, h\}) .
$$

Therefore $\mathcal{N}$ is a subalgebra of $\mathcal{O}_{M}$ • with respect to (2) and this Poisson algebra structure induces a Poisson algebra structure on $\mathcal{N} / \mathcal{N} \cap \mathcal{I}_{M}$.

Remark 1. The Poisson structure constructed in [1] corresponds to the case when $\phi(x)$ is monic of degree $d+2$ and has only simple roots. With such $\phi(x)$, the Poisson structure $(2)$ on $M_{r}\left(S_{d+1}\right)$ is equivalent to the canonical Poisson structure on $M_{r}(\mathbb{C})^{d+2}$, on which the discussion in [1] is based. See Appendix A for the explicit correspondence.

\subsection{Multi-Hamiltonian structure}

We define a family of Poisson structures on $\mathcal{M}$ :

Definition 2. For $\phi(x) \in S_{d+2},\{\cdot, \cdot\}_{\phi}: \mathcal{O}_{\mathcal{M}} \times \mathcal{O}_{\mathcal{M}} \rightarrow \mathcal{O}_{\mathcal{M}}$ denotes the Poisson structure on $\mathcal{M}$ obtained in Proposition 1. For $0 \leq i \leq d+2$, we write $\{\cdot, \cdot\}_{i}:=\{\cdot, \cdot\}_{\phi}$ with $\phi(x)=x^{i}$.

By construction, the Poisson structures are compatible:

$$
\{\cdot, \cdot\}_{c_{1} \phi_{1}+c_{2} \phi_{2}}=c_{1}\{\cdot, \cdot\}_{\phi_{1}}+c_{2}\{\cdot, \cdot\}_{\phi_{2}} \quad\left(c_{1}, c_{2} \in \mathbb{C}, \phi_{1}(x), \phi_{2}(x) \in S_{d+2}\right) .
$$

Define the $P G L_{r}(\mathbb{C})$-invariant functions $H_{i}^{(k)}(1 \leq k \leq r, 0 \leq i \leq k d)$ on $M_{r}\left(S_{d}\right)$ by

$$
\frac{1}{k} \operatorname{Tr} A(x)^{k}=\sum_{i=0}^{k d} H_{i}^{(k)} x^{i} \quad \text { for } \quad A(x) \in M_{r}\left(S_{d}\right) .
$$

Lemma 1. (Cf. [1, proposition in (5.2)].) The Hamiltonian vector field of $H_{j}^{(k)}(1 \leq k \leq r$, $0 \leq j \leq d k)$ with respect to the Poisson structure $\{\cdot, \cdot\}_{\phi}$ is related to the vector fields (1) as follows:

$$
\left\{H_{j}^{(k)}, *\right\}_{\phi}=\sum_{i=0}^{\min (j, d+2)} \sigma_{i} \eta_{*} Y_{j-i}^{(k-1)}
$$

In particular, $H_{j}^{(1)}(0 \leq j \leq d)$ are Casimir functions.

Proof. By direct calculation, we can show that for each $\phi(x) \in S_{d+2}$ and $k \geq 1$, the Hamiltonian vector field of $\frac{1}{k} \operatorname{Tr} A(a)^{k}\left(a \in \mathbb{P}^{1}\right)$ on $M_{r}\left(S_{d+1}\right)$ with respect to the Poisson structure (2) is

$$
\frac{\phi(a)}{x-a}\left[A(x), A(a)^{k-1}\right]
$$

It is easy to show that this is tangent to $M_{r}\left(S_{d}\right)$ and that its restriction to $M_{r}\left(S_{d}\right)$ is

$$
\frac{\phi(a)}{x-a}\left[A(x), A(a)^{k-1}\right]=\sum_{i=0}^{d+2} \sum_{j=0}^{d(k-1)-1} \sigma_{i} a^{i+j} Y_{j}^{(k-1)}(A(x)) .
$$

By Proposition 1, the corresponding Hamiltonian vector field is given by a push forward of (3) by $\eta$. Comparing the coefficients of powers of $a$, we obtain the lemma. 
Theorem 1. (i) Each $\eta_{*} Y_{j}^{(k)}$ is a multi-Hamiltonian vector field with respect to the Poisson structures $\{\cdot, \cdot\}_{i}(i=0, \ldots, d+2)$ :

$$
\eta_{*} Y_{j}^{(k)}=\left\{H_{j}^{(k+1)}, *\right\}_{0}=\left\{H_{j+i}^{(k+1)}, *\right\}_{i}
$$

for $1 \leq k \leq r-1$ and $0 \leq j \leq k d-2$.

(ii) With respect to $\{\cdot, \cdot\}_{i}(0 \leq i \leq d+2), H_{0}^{(k)}, \ldots, H_{i-1}^{(k)}$ and $H_{d(k-1)+i-1}^{(k)}, \ldots, H_{d k}^{(k)}(1 \leq k \leq r)$ are Casimir functions.

Proof. By Lemma 1, we obtain

$$
\begin{array}{ll}
\left\{H_{j}^{(k+1)}, *\right\}_{i}=\eta_{*} Y_{j-i}^{(k)} & \text { for } \quad i \leq j \leq d k+i-1, \\
\left\{H_{j}^{(k+1)}, *\right\}_{i}=0 & \text { for } \quad 0 \leq j \leq i-1 \quad \text { and } \quad d k+i \leq j \leq d(k+1) .
\end{array}
$$

Moreover, $\eta_{*} Y_{d k-1}^{(k)}=0$ since $Y_{d k-1}^{(k)}$ is tangent to $P G L_{r}(\mathbb{C})$-orbits by the definition (1). This proves the theorem.

\subsection{Poisson structure for representatives of $\mathcal{M}(2, d)$}

In this subsection, we explicitly write down the Poisson structure $\{\cdot, \cdot\}_{\phi}$ in the case of $r=2$. We also discuss how this is related to the known Poisson structures on the Mumford system.

Consider the subspace $\mathcal{S}_{\infty} \subset M_{2}\left(S_{d}\right)$ defined by

$$
\mathcal{S}_{\infty}=\left\{S(x)=\left(\begin{array}{cc}
v_{d} & 0 \\
1 & 0
\end{array}\right) x^{d}+\left(\begin{array}{cc}
v_{d-1} & u_{d-1} \\
w_{d-1} & 0
\end{array}\right) x^{d-1}+\left(\begin{array}{cc}
v_{d-2} & u_{d-2} \\
w_{d-2} & t_{d-2}
\end{array}\right) x^{d-2}+\cdots \mid u_{d-1} \neq 0\right\} .
$$

In Section 4, we will see that $\mathcal{S}_{\infty}$ is a space of representatives for $M_{\infty}$ which is an open subset of

$$
M_{2 d}=\left\{A(x) \in M_{2}\left(S_{d}\right) \mid H_{2 d}^{(2)}=0\right\} .
$$

Lemma 2. If $\phi(x) \in S_{d+1}$, (2) induces a Poisson structure on $\mathcal{S}_{\infty}$.

Proof. In this proof we write $M$ for $M_{2}\left(S_{d}\right)$. By Proposition 1, we have the Poisson algebra structure $\{\cdot, \cdot\}_{\phi}$ on the sheaf $\mathcal{O}_{M}^{G}$. Moreover, $H_{2 d}^{(2)}$ is its Casimir function since $\operatorname{deg} \phi \leq d+1$ (Theorem 1). Therefore the Poisson algebra structure induces that on $\mathcal{O}_{M}^{G} / \mathcal{O}_{M}^{G} \cap \mathcal{I}_{M_{2 d}}$, where $\mathcal{I}_{M_{2 d}}$ is the ideal sheaf of $M_{2 d}$ in $M=M_{2}\left(S_{d}\right)$. Thus (2) induces the Poisson structure on $M_{\infty} / G \cong \mathcal{S}_{\infty}$.

By a direct calculation (cf. proof of Proposition 4), we obtain the next result.

Proposition 2. For $\phi(x)=\sigma_{d+1} x^{d+1}+\cdots+\sigma_{0} \in S_{d+1}$, the Poisson structure $\{\cdot, \cdot\}_{\phi}$ is written as follows

$$
\begin{aligned}
\{S(x) \stackrel{\otimes}{,} S(y)\}_{\phi}= & \phi(y)\left[r(x, y), S(x) \otimes \mathbb{I}_{2}\right]-\phi(x)\left[\bar{r}(x, y), \mathbb{I}_{2} \otimes S(y)\right] \\
& +\left[K(x, y), S(x) \otimes \mathbb{I}_{2}\right]-\left[\bar{K}(x, y), \mathbb{I}_{2} \otimes S(y)\right]
\end{aligned}
$$

where

$$
\{S(x) \stackrel{\otimes}{,} S(y)\}_{\phi}=\sum_{1 \leq i, j, k, l \leq r} E_{i j} \otimes E_{k l}\left\{S_{i j}(x), S_{k l}(y)\right\}_{\phi}
$$


and

$$
\begin{aligned}
& r(x, y)=\frac{1}{x-y} \mathbb{P}_{2}+\frac{1}{u_{d-1}}\left(\begin{array}{ll}
v_{d} & 0 \\
1 & 0
\end{array}\right) \otimes\left(\begin{array}{ll}
0 & 0 \\
1 & 0
\end{array}\right), \\
& \bar{r}(x, y)=\mathbb{P}_{2} \cdot r(y, x) \cdot \mathbb{P}_{2}, \\
& K(x, y)=\frac{1}{u_{d-1}}\left(\begin{array}{cc}
y v_{d} \sigma_{d+1} & -u_{d-1} \sigma_{d+1} \\
-\left(w_{d-1}-y\right) \sigma_{d+1}+\sigma_{d} & 0
\end{array}\right) \otimes\left(\begin{array}{cc}
0 & S_{12}(y) \\
-S_{21}(y) & 0
\end{array}\right), \\
& \bar{K}(x, y)=\mathbb{P}_{2} \cdot K(y, x) \cdot \mathbb{P}_{2}, \quad \mathbb{P}_{2}=\sum_{1 \leq i, j \leq 2} E_{i j} \otimes E_{j i} .
\end{aligned}
$$

We write $F_{j}^{(1)}(j=0, \ldots, d-2)$ for the vector field on $\mathcal{S}_{\infty}$ induced by $\eta_{*} Y_{j}^{(1)}$. As a consequence of Theorem 1 and Proposition 2 we obtain

Corollary 1. Each $F_{j}^{(1)}(j=0, \ldots, d-2)$ is the multi-Hamiltonian vector field with respect to the Poisson structure (4). They are written as the Lax form:

$$
\sum_{j=0}^{d-2} y^{j} F_{j}^{(1)}(S(x))=\frac{1}{y^{i}}\left\{H^{(2)}(y), S(x)\right\}_{i}=\left[S(x), \frac{1}{x-y} S(y)+\frac{S_{12}(y)}{u_{d-1}}\left(\begin{array}{cc}
v_{d} & 0 \\
1 & 0
\end{array}\right)\right],
$$

for $i=0, \ldots, d+1$.

Now we derive a Poisson structure of the Mumford system from (4). The phase space $\mathcal{S}_{\text {Mum }}$ of the Mumford system is the subspace of $\mathcal{S}_{\infty}$ defined as

$$
\mathcal{S}_{\mathrm{Mum}}=\left\{S(x) \in \mathcal{S}_{\infty} \mid \operatorname{Tr} S(x)=0, u_{d-1}=1\right\} .
$$

Lemma 3. (4) induces a Poisson structure on $\mathcal{S}_{\mathrm{Mum}}$ if $\sigma_{d+1}=0$.

Proof. When $\operatorname{deg} \phi(x) \leq d, H_{2 d-1}^{(2)}=u_{d-1}$ is a Casimir of $\{\cdot, \cdot\}_{\phi}$ by Theorem 1 . Therefore (4) induces a Poisson structure on $\mathcal{S}_{\mathrm{Mum}}$.

This is the same as the Poisson structure in $[8, \S 5.1]$. The Poisson structures in [3, (4)] and [7] correspond to the case $\sigma_{d+1}=\sigma_{d}=0$ and the case $\phi(x)=x$ respectively. The formula (5) reduces to the Lax form for the Mumford system [3, (7)].

\section{Multi-Hamiltonian structure on the Beauville-Vanhaecke system}

\subsection{The Beauville-Vanhaecke system}

Following [5], we define the set $M^{\prime}(r, d)$ and the group $G_{r}$ as

$$
\begin{aligned}
& M^{\prime}(r, d)=\left\{A(x) \in M_{r}(\mathbb{C}[x]) \mid \begin{array}{c}
A(x)_{11} \in S_{d}, \quad A(x)_{1 j} \in S_{d+1}, \quad \\
A(x)_{i 1} \in S_{d-1}, \quad A(x)_{i j} \in S_{d},
\end{array} \quad(2 \leq i, j \leq r)\right\}, \\
& G_{r}=\left\{g(x)=\left(\begin{array}{cc}
1 & { }^{t} \vec{b}_{1} x+{ }^{t} \vec{b}_{0} \\
0 & B
\end{array}\right) \mid B \in G L_{r-1}(\mathbb{C}), \quad \vec{b}_{1}, \vec{b}_{0} \in \mathbb{C}^{r-1}\right\} .
\end{aligned}
$$

Here we use the notation such as $\vec{b}$ for a column vector and ${ }^{t} \vec{b}$ for a row vector. The group $G_{r}$ acts on $M^{\prime}(r, d)$ by conjugation. Let $\psi: M^{\prime}(r, d) \rightarrow V(r, d)$ be the map $\psi(A(x))=\operatorname{det}(y \mathbb{I}-A(x))$ and define

$$
M^{\prime}(r, d)_{i r}=\left\{A(x) \in M^{\prime}(r, d) \mid \text { the spectral curve } C_{\psi(A(x))} \text { is irreducible }\right\} .
$$


The $G_{r}$-action is free on $M_{r}\left(S_{d}\right)_{i r}\left[5\right.$, Lemma 2.6]. Let $\eta^{\prime}: M^{\prime}(r, d)_{i r} \rightarrow M^{\prime}(r, d)_{i r} / G_{r}$ be the quotient map. The phase space of the Beauville-Vanhaecke system is $\mathcal{M}^{\prime}(r, d)=M^{\prime}(r, d)_{i r} / G_{r}$. It was shown that if $P \in V(r, d)$ defines a smooth spectral curve $C_{P}$, then $\psi^{-1}(P) / G_{r}$ is isomorphic to the complement of the intersection of $r$-translates of the theta divisor in $\operatorname{Pic}^{g}\left(C_{P}\right)$ [5, Theorem 2.8].

Define the vector fields $Y_{i}^{(k)}$ on $M^{\prime}(r, d)$ by

$$
\sum_{i=0}^{k d} a^{i} Y_{i}^{(k)}(A(x))=\frac{1}{x-a}\left[A(x), A(a)^{k}\right], \quad \text { for } \quad k=1, \ldots, r-1 .
$$

It was shown that $\eta_{*}^{\prime} Y_{i}^{(k)}$ generate the $g$-dimensional space of translation invariant vector fields on $\operatorname{Pic}^{g}\left(C_{P}\right)^{1}$.

\subsection{Poisson structure}

We equip $\mathcal{M}^{\prime}(r, d)$ with a family of Poisson structures, extending the results in $[5, \S 3]$. The key idea is that (2) induces the Poisson structure on $\mathcal{M}^{\prime}(r, d)$ as in the case of the Beauville system. However, due to the technical difficulties arising from the $G_{r}$-action, we need a modification of the argument.

We use the following shorthand notations:

$$
M^{\prime}=M^{\prime}(r, d), \quad M_{i r}^{\prime}=M^{\prime}(r, d)_{i r}, \quad \mathcal{M}^{\prime}=\mathcal{M}^{\prime}(r, d) .
$$

Let us write $A(x) \in M^{\bullet}$ and $A(x) \in M^{\prime}$ as

$$
A(x)=\left(\begin{array}{cc}
v(x) & { }^{t} \vec{w}(x) \\
\vec{u}(x) & t(x)
\end{array}\right)
$$

where

$$
\begin{aligned}
& v(x)=A_{11}(x), \quad{ }^{t} \vec{w}(x)=\left(A_{12}(x), \ldots, A_{1 r}(x)\right), \\
& \vec{u}(x)={ }^{t}\left(A_{21}(x), \ldots, A_{r 1}(x)\right), \quad t(x)=\left(A_{i j}(x)\right)_{2 \leq i, j \leq r} .
\end{aligned}
$$

Let $\iota^{\prime}: M^{\prime} \hookrightarrow M^{\bullet}$ be the closed immersion and $\pi^{\prime}: M^{\bullet} \rightarrow M^{\prime}$ be the surjection:

$$
A(x)=\left(\begin{array}{ll}
\sum_{k=0}^{d+1} v_{k} x^{k} & \sum_{k=0}^{d+1} t_{w_{k}} x^{k} \\
\sum_{k=0}^{d+1} \vec{u}_{k} x^{k} & \sum_{k=0}^{d+1} t_{k} x^{k}
\end{array}\right) \mapsto\left(\begin{array}{ll}
\sum_{k=0}^{d} v_{k} x^{k} & \sum_{k=0}^{d+1} t_{w_{k}} x^{k} \\
\sum_{k=0}^{d-1} \vec{u}_{k} x^{k} & \sum_{k=0}^{d} t_{k} x^{k}
\end{array}\right) .
$$

Note that $\pi^{\prime} \circ \iota^{\prime}=i d_{M^{\prime}}$. Let $\gamma$ be the composition of the morphisms:

$$
\operatorname{Hom}\left(\left(\mathcal{O}_{M} \bullet\right)^{2}, \mathcal{O}_{M} \bullet\right) \stackrel{\pi_{*}^{\prime}}{\rightarrow} \operatorname{Hom}\left(\left(\pi_{*}^{\prime} \mathcal{O}_{M} \bullet\right)^{2}, \pi_{*}^{\prime} \mathcal{O}_{M} \bullet\right) \rightarrow \operatorname{Hom}\left(\left(\mathcal{O}_{M^{\prime}}\right)^{2}, \mathcal{O}_{M^{\prime}}\right),
$$

where the second morphism is given by

$$
\Phi \mapsto\left[\left(\mathcal{O}_{M^{\prime}}\right)^{2} \stackrel{\left(\pi^{\prime \#}\right)^{2}}{\rightarrow}\left(\pi_{*}^{\prime} \mathcal{O}_{M} \bullet\right)^{2} \stackrel{\Phi}{\rightarrow} \pi_{*}^{\prime} \mathcal{O}_{M} \bullet \stackrel{\pi_{*}^{\prime}\left(\iota^{\prime \#}\right)}{\rightarrow} \pi_{*}^{\prime} \iota_{*}^{\prime} \mathcal{O}_{M^{\prime}}=O_{M^{\prime}}\right]
$$

\footnotetext{
${ }^{1}$ Although $Y_{i}^{(k)}$ is not $G_{r}$-invariant, $\eta_{*}^{\prime} Y_{i}^{(k)}$ is well-defined because the difference between $g(x)_{*} Y_{i}^{(k)}$ and $Y_{i}^{(k)}$ is tangent to $G_{r}$-orbits [5, Lemma 3.2].
} 
We define $\{\cdot, \cdot\}^{B V} \in \operatorname{Hom}\left(\left(\mathcal{O}_{M^{\prime}}\right)^{2}, \mathcal{O}_{M^{\prime}}\right)$ to be the image of (2) by $\gamma$. For the coordinate functions $A_{i j ; k}$ of $M^{\prime}$, it is written explicitly as

$$
\left\{A_{i j}(x), A_{k l}(y)\right\}^{B V}=\left[\delta_{i, l} \frac{A_{k j}(x) \phi(y)-\phi(x) A_{k j}(y)}{x-y}-\delta_{k, j} \frac{A_{i l}(x) \phi(y)-\phi(x) A_{i l}(y)}{x-y}\right]_{\leq d_{i j}, \leq d_{k l}}
$$

where $[\cdot]_{\leq d_{i j}, \leq d_{k l}}$ means taking the terms whose degree in $x$ is smaller or equal to $d_{i j}$ and whose degree in $y$ is smaller or equal to $d_{k l}$. Here $d_{i j}=d, d_{1 j}=d+1, d_{i 1}=d-1$ for $2 \leq i, j \leq r$ and $d_{11}=d$.

Proposition 3. The sheaf $\mathcal{O}_{M^{\prime}}^{G_{r}}$ of $G_{r}$-invariant regular functions on $M^{\prime}$ is closed with respect to (7). Moreover, (7) is a Poisson algebra structure on $\mathcal{O}_{M^{\prime}}^{G_{r}}$.

The proof is delegated to Subsection 3.3. As an immediate consequence of this proposition, we obtain the Poisson algebra structure on $\mathcal{O}_{M_{i r}^{\prime}}^{G_{r}}$, which is equivalent to the Poisson structure on $\mathcal{M}^{\prime}$.

Remark 2. The Poisson structure constructed in $[5, \S 3]$ corresponds to the case where $\phi(x)$ is monic of degree $d+2$ and has only simple roots.

\subsection{Proof of Proposition 3}

We prove Proposition 3 in the cases of $\operatorname{deg} \phi(x)=d+2, \operatorname{deg} \phi(x)=d+1$ and $\operatorname{deg} \phi(x) \leq d$ separately.

The case of $\operatorname{deg} \phi(x)=d+2$ : We equip $M^{\bullet}$ with the Poisson structure (2). We extend the $G_{r^{-}}$action on $M^{\prime}$ to $M^{\bullet}$ as follows ${ }^{2}$ :

$$
G_{r} \ni g(x): A(x) \mapsto \tilde{A}(x),
$$

where $\tilde{A}(x) \in M_{r}\left(S_{d+1}\right)$ is the matrix uniquely determined by

$$
g(x)^{-1} A(x) g(x)=\tilde{A}(x)+\phi(x) \hat{A}(x), \quad \hat{A}(x) \in M_{r}\left(S_{1}\right) .
$$

By direct calculation, we can show that the Poisson structure (2) is invariant with respect to this $G_{r}$-action.

Let $\mathcal{I}_{M^{\prime}}$ be the ideal sheaf of $\iota^{\prime}$, and set $\mathcal{N}^{\prime}:=\alpha^{\prime-1}\left(\iota_{*}^{\prime} \mathcal{O}_{M^{\prime}}^{G_{r}}\right) \subset \mathcal{O}_{M} \bullet$ by writing $\alpha^{\prime}$ for the natural projection $\mathcal{O}_{M} \bullet \rightarrow \mathcal{O}_{M} \bullet / \mathcal{I}_{M^{\prime}}=\iota_{*}^{\prime} \mathcal{O}_{M^{\prime}}$.

Lemma 4. (1) $\mathcal{N}^{\prime}$ is a Poisson subalgebra of $\mathcal{O}_{M} \bullet$

(2) The Poisson algebra structure of (1) induces that on $\mathcal{N}^{\prime} / \mathcal{N}^{\prime} \cap \mathcal{I}_{M^{\prime}}$, hence on $\mathcal{O}_{M^{\prime}}^{G_{r}}$. Moreover, it is given by $(7)$.

Proof. Note that for $f \in \mathcal{O}_{M} \bullet(U)$ where $U$ is any open subset,

$$
\begin{aligned}
& \sum_{i=1}^{r}\left\{A_{i i ; d+1}, f\right\}=0, \\
& \left\{A_{i j ; d+1}, f\right\}=-\sigma_{d+2} X_{E_{j i}} f \quad(2 \leq i, j \leq r), \\
& \left\{A_{i 1 ; d+1}, f\right\}=-\sigma_{d+2} X_{E_{1 i}} f, \quad\left\{A_{i 1 ; d}, f\right\}=-\sigma_{d+2} X_{E_{1 i}^{\prime}} f-\sigma_{d+1} X_{E_{1 i}} f \quad(2 \leq i \leq r) .
\end{aligned}
$$

\footnotetext{
${ }^{2}$ In the case $\phi(x)$ has only simple roots, this action is the same as the one used in [5]. See Appendix B for a proof.
} 
Here $X_{E_{j i}}, X_{E_{1 i}}, X_{E_{1 i}^{\prime}}$ are the vector fields generating the infinitesimal actions corresponding to $E_{j i}, E_{1 i}, E_{1 i}^{\prime}=x E_{1 i} \in \operatorname{Lie} G_{r}$ :

$$
\begin{aligned}
& X_{E_{i j}}(A(x))=\left[A(x), E_{i j}\right] \quad(2 \leq i, j \leq r), \\
& X_{E_{1 j}}(A(x))=\left[A(x), E_{1 j}\right] \quad(2 \leq j \leq r), \\
& X_{E_{1 j}^{\prime}}(A(x))=\left[x A(x)-\frac{\sigma_{d+1}}{\sigma_{d+2}} A^{d+1}, E_{1 j}\right] \quad(2 \leq j \leq r) \text {. }
\end{aligned}
$$

By the same argument as that of Proposition 1, we can show that $\mathcal{N}^{\prime}$ is a Poisson subalgebra of $\mathcal{O}_{M^{\prime}}$ and that this induces a Poisson algebra structure on $\mathcal{N}^{\prime} / \mathcal{N}^{\prime} \cap I_{M^{\prime}}$, hence on $\mathcal{O}_{M^{\prime}}^{G_{r}}$. By construction, the Poisson algebra structure on $\mathcal{O}_{M^{\prime}}^{G_{r}}$ coincides with the restriction of (7) to $\mathcal{O}_{M^{\prime}}^{G_{r}}$.

The Case of $\operatorname{deg} \phi(x)=d+1$ : For an open subset $U$ of $M^{\prime}$, a function $F \in \mathcal{O}_{M^{\prime}}^{G_{r}}(U)$ is characterized by the condition $X_{E_{i j}} F=X_{E_{1 j}} F=X_{E_{1 j}^{\prime}} F=0(2 \leq i, j \leq r)$ where $X_{E_{i j}}, X_{E_{1 j}}$, $X_{E_{1 j}^{\prime}}$ are the infinitesimal action on $M^{\prime}$ corresponding to $E_{i j}, E_{1 j}, E_{1 j}^{\prime} \in \operatorname{Lie} G_{r}$ :

$$
\begin{aligned}
& X_{E_{i j}}(A(x))=\left[A(x), E_{i j}\right] \quad(2 \leq i, j \leq r), \\
& X_{E_{1 j}}(A(x))=\left[A(x), E_{1 j}\right] \quad(2 \leq j \leq r), \\
& X_{E_{1 j}^{\prime}}(A(x))=\left[x A(x), E_{1 j}\right] \quad(2 \leq j \leq r) \text {. }
\end{aligned}
$$

Using this fact, we can show that $\mathcal{O}_{M^{\prime}}^{G_{r}}$ is closed with respect to $\{\cdot, \cdot\}^{B V}$. We can also show that the Jacobi identity holds on $\mathcal{O}_{M^{\prime}}^{G_{r}}$ although it does not on $\mathcal{O}_{M^{\prime}}$. Thus $\{\cdot, \cdot\}^{B V}$ is a Poisson algebra structure on $\mathcal{O}_{M^{\prime}}^{G_{r}}$.

The Case of $\operatorname{deg} \phi(x) \leq d$ : When $\operatorname{deg} \phi(x) \leq d,\{\cdot, \cdot\}^{B V}$ satisfies the Jacobi identity on $\mathcal{O}_{M^{\prime}}$ since it gives $\mathcal{O}_{M^{\prime}}$ a Poisson subalgebra structure. By using the action (10) of Lie $G_{r}$ on $M^{\prime}$, we can show that $\mathcal{O}_{M^{\prime}}^{G_{r}}$ is closed with respect to $\{\cdot, \cdot\}^{B V}$ as in the case of $\operatorname{deg} \phi(x)=d+1$. Therefore $\{\cdot, \cdot\}^{B V}$ is a Poisson algebra structure on $\mathcal{O}_{M^{\prime}}^{G_{r}}$

\subsection{Multi-Hamiltonian structure}

Definition 3. For $\phi(x) \in S_{d+2},\{\cdot, \cdot\}_{\phi}^{\prime}: \mathcal{O}_{\mathcal{M}^{\prime}} \times \mathcal{O}_{\mathcal{M}^{\prime}} \rightarrow \mathcal{O}_{\mathcal{M}^{\prime}}$ denotes the Poisson structure defined in Proposition 3. For $0 \leq i \leq d+2$, we write $\{\cdot, \cdot\}_{i}^{\prime}:=\{\cdot, \cdot\}_{\phi}^{\prime}$ with $\phi(x)=x^{i}$.

By construction, these Poisson structures are compatible in the sense of Subsection 2.3.

Define the $G_{r}$-invariant functions $H_{i}^{(k)}(1 \leq k \leq r, 0 \leq i \leq k d)$ on $M^{\prime}(r, d)$ by

$$
\frac{1}{k} \operatorname{Tr} A(x)^{k}=\sum_{i=0}^{k d} H_{i}^{(k)} x^{i} \quad \text { for } \quad A(x) \in M^{\prime}(r, d) .
$$

Lemma 5. (Cf. [5, Lemma 3.10].) The Hamiltonian vector field of $H_{j}^{(k)}(1 \leq k \leq r, 0 \leq i \leq d k)$ is related to the vector fields (6) as

$$
\left\{H_{j}^{(k)}, *\right\}_{\phi}^{\prime}=\sum_{i=0}^{\min (j, d+2)} \sigma_{i} \eta_{*} Y_{j-i}^{(k-1)}
$$

In particular, $H_{j}^{(1)}(0 \leq j \leq d)$ are Casimir functions of $\{\cdot, \cdot\}_{\phi}^{\prime}$.

This lemma can be proved in the same way as Lemma 1 using Proposition 3. From Lemma 5, we obtain the following theorem similar to Theorem 1. 
Theorem 2. (i) Each $\eta_{*} Y_{j}^{(k)}$ is a multi-Hamiltonian vector field with respect to the Poisson structures $\{\cdot, \cdot\}_{i}^{\prime}(i=0, \ldots, d+2)$ :

$$
\eta_{*} Y_{j}^{(k)}=\left\{H_{j}^{k+1}, *\right\}_{0}^{\prime}=\left\{H_{j+i}^{k+1}, *\right\}_{i}^{\prime}
$$

for $1 \leq k \leq r-1$ and $0 \leq j \leq k d-2$.

(ii) With respect to $\{\cdot, \cdot\}_{i}^{\prime}(0 \leq i \leq d+2), H_{0}^{(k)}, \ldots, H_{i-1}^{(k)}$ and $H_{d(k-1)+i-1}^{(k)}, \ldots, H_{d k}^{(k)}(1 \leq k \leq r)$ are Casimir functions.

\subsection{Poisson structure for representatives of $\mathcal{M}^{\prime}(2, d)$}

We present the Poisson structure $\{\cdot, \cdot\}_{\phi}^{\prime}$ in the case $r=2$ on the space of representatives $\mathcal{S}_{\infty}^{\prime}$ of $\mathcal{M}^{\prime}(2, d)$ constructed in $[5, \S 4]$.

The space $\mathcal{S}_{\infty}^{\prime}$ is written as

$$
\mathcal{S}_{\infty}^{\prime}=\left\{S(x)=\left(\begin{array}{cc}
0 & w_{d+1} \\
0 & 0
\end{array}\right) x^{d+1}+\left(\begin{array}{cc}
v_{d} & w_{d} \\
0 & 0
\end{array}\right) x^{d}+\left(\begin{array}{cc}
v_{d-1} & w_{d-1} \\
1 & 0
\end{array}\right) x^{d-1}+\text { lower terms in } x\right\} .
$$

We obtain the following result by a direct calculation.

Proposition 4. For $\phi(x)=\sigma_{d+2} x^{d+2}+\sigma_{d+1} x^{d+1}+\cdots+\sigma_{0}$, the Poisson bracket $\{\cdot, \cdot\}_{\phi}^{\prime}$ is written as follows:

$$
\begin{aligned}
\{S(x) \stackrel{\otimes}{,} S(y)\}_{\phi}^{\prime}= & \phi(y)\left[r(x, y), S(x) \otimes \mathbb{I}_{2}\right]-\phi(x)\left[\bar{r}(x, y), \mathbb{I}_{2} \otimes S(y)\right] \\
& +B(x, y)\left[K(x, y), S(x) \otimes \mathbb{I}_{2}\right]-B(x, y)\left[\bar{K}(x, y), \mathbb{I}_{2} \otimes S(y)\right],
\end{aligned}
$$

where

$$
\begin{aligned}
& r(x, y)=\frac{1}{x-y} \mathbb{P}_{2}+\left(\begin{array}{cc}
v_{d} & A(x+y) \\
0 & 0
\end{array}\right) \otimes\left(\begin{array}{ll}
0 & 1 \\
0 & 0
\end{array}\right), \\
& K(x, y)=\left(\begin{array}{cc}
0 & B(x, y) \\
0 & 0
\end{array}\right) \otimes\left(\begin{array}{cc}
0 & -S_{12}(y) \\
S_{21}(y) & 0
\end{array}\right), \quad \bar{K}(x, y)=\mathbb{P}_{2} \cdot r(y, x) \cdot \mathbb{P}_{2}, \\
& A(x)=w_{d+1}\left(x-u_{d-2}\right)+w_{d}, \\
& B(x, y)=\sigma_{d+2}\left(x^{2}+y^{2}+x y+\left(u_{d-2}-x-y\right) u_{d-2}-u_{d-3}\right)+\sigma_{d+1}\left(u_{d-2}-x-y\right)+\sigma_{d} .
\end{aligned}
$$

Proof. In this proof, we write $A(x) \in M^{\prime}$ as

$$
A(x)=\left(\begin{array}{ll}
\sum_{i=0}^{d} \tilde{v}_{i} x^{i} & \sum_{i=0}^{d+1} \tilde{w}_{i} x^{i} \\
\sum_{i=0}^{d-1} \tilde{u}_{i} x^{i} & \sum_{i=0}^{d} \tilde{t}_{i} x^{i}
\end{array}\right)
$$

and regard $\tilde{v}_{i}(0 \leq i \leq d)$, $\tilde{w}_{i}(0 \leq i \leq d+1), \tilde{u}_{i}(0 \leq i \leq d-1), \tilde{t}_{i}(0 \leq i \leq d)$ as coordinate functions of $M^{\prime}$. (Here we use ${ }^{\sim}$ to distinguish from $v_{i}, w_{i}, u_{i}, t_{i}$ which we use as coordinates of $\mathcal{S}_{\infty}^{\prime}$.) The bracket $\{\cdot, \cdot\}^{B V}(7)$ among $\tilde{v}_{i}, \ldots, \tilde{t}_{i}$ are explicitly written as follows:

$$
\begin{aligned}
\{\tilde{v}(x), \tilde{v}(y)\}= & \{\tilde{u}(x), \tilde{u}(y)\}=\{\tilde{w}(x), \tilde{w}(y)\}=\{\tilde{t}(x), \tilde{t}(y)\}=\{\tilde{v}(x), \tilde{t}(y)\}=0, \\
\{\tilde{v}(x), \tilde{u}(y)\}= & -\{\tilde{t}(x), \tilde{u}(y)\}=\frac{\tilde{u}(x) \phi(y)-\phi(x) \tilde{u}(y)}{x-y} \\
& +\sigma_{d+2} \tilde{u}(y) x^{d+1}+\sigma_{d+2} \tilde{u}(x) y^{d+1}-\left(\sigma_{d+1}-\sigma_{d+2} x\right) \tilde{u}(x) y^{d},
\end{aligned}
$$




$$
\begin{aligned}
\{\tilde{v}(x), \tilde{w}(y)\}= & -\{\tilde{t}(x), \tilde{w}(y)\}=-\frac{\tilde{w}(x) \phi(y)-\phi(x) \tilde{w}(y)}{x-y}-\sigma_{d+2} \tilde{w}(y) x^{d+1}, \\
\{\tilde{u}(x), \tilde{w}(y)\}= & \frac{(\tilde{v}(x)-\tilde{t}(x)) \phi(y)-\phi(x)(\tilde{v}(y)-\tilde{t}(y))}{x-y} \\
& +\sigma_{d+2}(\tilde{v}(y)-\tilde{t}(y)) x^{d+1}-\left(\sigma_{d+1}-\sigma_{d+2} y\right)(\tilde{v}(y)-\tilde{t}(y)) x^{d},
\end{aligned}
$$

where $\tilde{v}(x):=\sum_{i=0}^{d} \tilde{v}_{i} x^{i}$ and so on.

Similarly let $v_{i}(0 \leq i \leq d), w_{i}(0 \leq i \leq d+1), u_{i}(0 \leq i \leq d-2), t_{i}(0 \leq i \leq d-2)$ denote coordinate functions of $\mathcal{S}_{\infty}^{\prime}$. Let $\left(b_{1}, b_{0}, c\right) \in \mathbb{C}^{2} \times \mathbb{C}^{*}$ be the following coordinate functions of $G_{r}$ :

$$
G_{r} \ni\left(\begin{array}{cc}
1 & b_{1} x+b_{0} \\
0 & c
\end{array}\right)
$$

In the neighborhood of $\mathcal{S}_{\infty}^{\prime},\left(v_{i}(0 \leq i \leq d), w_{i}(0 \leq i \leq d+1), u_{i}(0 \leq i \leq d-2), t_{i}\right.$ $\left.(0 \leq i \leq d-2), b_{0}, b_{1}, c\right)$ forms a local coordinate system of $M^{\prime}$. The transformation between the two coordinate systems is given by

$$
\left(\begin{array}{cc}
\tilde{v}(x) & \tilde{w}(x) \\
\tilde{u}(x) & \tilde{t}(x)
\end{array}\right)=\left(\begin{array}{cc}
1 & b_{1} x+b_{0} \\
0 & c
\end{array}\right)^{-1}\left(\begin{array}{cc}
v(x) & w(x) \\
u(x) & t(x)
\end{array}\right)\left(\begin{array}{cc}
1 & b_{1} x+b_{1} \\
0 & c
\end{array}\right)
$$

Substituting the RHS of (13) into (12) and using the Leibniz rule, we obtain the system of equations for brackets among $\left(v(x), w(x), u(x), t(x), b_{0}, b_{1}, c\right)$. Solving this and restricting to $\mathcal{S}_{\infty}^{\prime}$ (i.e. setting $b_{0}=b_{1}=0, c=1$ ), we arrive at the result of Proposition 4 .

As in the case of the Beauville system, we write $F_{j}^{(1)}(j=0, \ldots, d-2)$ for the vector field on $\mathcal{S}_{\infty}^{\prime}$ induced by $\eta_{*} Y_{j}^{(1)}$. From Theorem 2 and Proposition 4 we obtain

Corollary 2. Each $F_{j}^{(1)}(j=0, \ldots, d-2)$ is the multi-Hamiltonian vector field with respect to the Poisson structure (11). They are written as the Lax form:

$$
\begin{aligned}
\sum_{j=0}^{d-2} y^{j} F_{j}^{(1)}(S(x)) & =\frac{1}{y^{i}}\left\{H^{(2)}(y), S(x)\right\}_{i} \\
& =\left[S(x), \frac{1}{x-y} S(y)+S_{21}(y)\left(\begin{array}{cc}
0 & A(x+y) \\
0 & -v_{d}
\end{array}\right)\right],
\end{aligned}
$$

for $i=0, \ldots, d+2$.

We remark that this Lax form already appeared in $[5,(4.9)]$ for general $r$.

In closing this subsection, we discuss the Poisson structure on the even Mumford system. The phase space of the even Mumford system is given by

$$
\left\{S(x) \in \mathcal{S}_{\infty}^{\prime} \mid \operatorname{Tr} S(x)=0, w_{d+1}=1\right\} .
$$

Lemma 6. If $\sigma_{d+2}=0$, (11) gives a Poisson structure on the phase space of the even Mumford system.

Proof. By Theorem 2, $w_{d+1}=H_{2 d}^{(2)}$ is Casimir of $\{\cdot, \cdot\}_{\phi}^{\prime}$ if $\operatorname{deg} \phi(x) \leq d+1$. Therefore in such a case, (11) induces a Poisson algebra structure on $\mathcal{O}\left(\mathcal{S}_{\infty}^{\prime}\right) / H_{2 d}^{(2)} \mathcal{O}\left(\mathcal{S}_{\infty}^{\prime}\right)$.

The Poisson structure in [3] corresponds to the case $\sigma_{d+2}=\sigma_{d+1}=\sigma_{d}=0$. 


\section{Representatives of the Beauville system}

First we introduce some notations. Let us define a subset $M_{\text {reg }}$ of $M_{r}(\mathbb{C})$ :

$$
M_{\mathrm{reg}}=\left\{A \in M_{r}(\mathbb{C}) \mid \operatorname{deg}(\text { the minimal polynomial of } A)=r\right\} .
$$

For $A \in M_{r}(\mathbb{C}), A \in M_{\text {reg }}$ is equivalent to the condition that only one Jordan block corresponds to each eigenvalue of $A$. For $A \in M_{\text {reg }}$, let $\alpha_{1}, \ldots, \alpha_{k}(k<r)$ be the distinct eigenvalues and $\nu_{1}, \ldots, \nu_{k}$ be the size of the corresponding Jordan blocks. Define the subspace of $\mathbb{C}^{r}$ as

$$
W_{\alpha_{i}: j}=\left\{\vec{u} \in \mathbb{C}^{r} \mid\left(A-\alpha_{i} \mathbb{I}_{r}\right)^{j} \vec{u}=0\right\}
$$

for $0 \leq i \leq k, j \in \mathbb{Z}_{\geq 0}$. The spaces $W_{\alpha_{i}: 1}$ and $W_{\alpha_{i}: \nu_{i}}$ are respectively the eigenspace and the generalized eigenspace of $A$. There is the filtration

$$
W_{\alpha_{i}: \nu_{i}} \supset W_{\alpha_{i}: \nu_{i}-1} \supset \cdots \supset W_{\alpha_{i}: 1} \supset W_{\alpha_{i}: 0}=\{\overrightarrow{0}\} .
$$

By the assumption of $A, \operatorname{dim}\left(W_{\alpha_{i}: j} / W_{\alpha_{i}: j-1}\right)=1$ for all $\alpha_{i}$ and $j=1, \ldots, \nu_{i}$. We fix a base $\vec{v}_{\alpha_{i}}(A)$ of $W_{\alpha_{i}: 1}$. Let $\Pi_{\alpha_{i}}$ be the projection map $\Pi_{\alpha_{i}}: \mathbb{C}^{r} \rightarrow W_{\alpha_{i}: \nu_{i}}$, and define

$$
V(A)=\left\{\vec{u} \in \mathbb{C}^{r} \mid \Pi_{\alpha_{i}}(\vec{u}) \notin W_{\alpha_{i}: \nu_{i}-1} \text { for } i=0, \ldots, k\right\} .
$$

Now we introduce the subspaces $M_{\infty}$ and $\mathcal{S}_{\infty}$ of $M_{r}\left(S_{d}\right)$ :

$$
\begin{aligned}
& M_{\infty}=\left\{A(x)=\sum_{k=0}^{d} A_{k} x^{k} \in M_{r}\left(S_{d}\right) \mid A_{d} \in M_{\mathrm{reg}}, \operatorname{det} A_{d}=0, A_{d-1} \vec{v}_{0}\left(A_{d}\right) \in V\left(A_{d}\right)\right\}, \\
& \mathcal{S}_{\infty}=\left\{A(x) \in M_{r}\left(S_{d}\right) \mid A(x)=\omega x^{d}+\rho x^{d-1}+\text { lower terms in } x, \omega \in \Omega, \rho \in \mathcal{T}\right\},
\end{aligned}
$$

where

$$
\begin{aligned}
& \Omega=\left\{\left(\begin{array}{cccc}
-\beta_{1} & \cdots & -\beta_{r-1} & 0 \\
1 & 0 & \cdots & 0 \\
\vdots & \ddots & \ddots & \vdots \\
0 & \cdots & 1 & 0
\end{array}\right) \in M_{r}(\mathbb{C}) \mid \beta_{1}, \cdots, \beta_{r-1} \in \mathbb{C}\right\} \\
& \mathcal{T}=\left\{\rho \in M_{r}(\mathbb{C}) \mid \rho_{1 r} \neq 0, \rho_{j r}=0 \text { for } j=2, \ldots, r\right\} .
\end{aligned}
$$

The main result of this section is as follows:

Proposition 5. (i) $\mathcal{S}_{\infty} \subset M_{\infty}$.

(ii) The action of $P G L_{r}(\mathbb{C})$ on $M_{\infty}$ induces an isomorphism $\mathcal{S}_{\infty} \times P G L_{r}(\mathbb{C}) \simeq M_{\infty}$. Thus the space $\mathcal{S}_{\infty}$ is a set of representatives of $M_{\infty} / P G L_{r}(\mathbb{C})$.

Remark 3. One can define $\mathcal{M}_{c}$ and $\mathcal{S}_{c}$ for $c \in \mathbb{C}$ as

$$
\begin{aligned}
& M_{c}=\left\{A(x) \in M_{r}\left(S_{d}\right) \mid A(c) \in M_{\mathrm{reg}}, \operatorname{det} A(c)=0, A^{\prime}(c) \vec{v}_{0}(A(c)) \in V(A(c))\right\}, \\
& \mathcal{S}_{c}=\left\{A(x) \in M_{r}\left(S_{d}\right) \mid A(x)=\omega+\rho(x-c)+\text { higher terms in }(x-c), \omega \in \Omega, \rho \in \mathcal{T}\right\} .
\end{aligned}
$$

Then Proposition 5 also holds for $\left(M_{c}, \mathcal{S}_{c}\right)$.

Let us recall the following lemmas on linear algebra. 


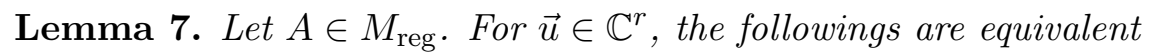

(i) $\vec{u} \in V(A)$;

(ii) ${ }^{t} \vec{v}_{\alpha}\left({ }^{t} A\right) \cdot \vec{u} \neq 0$ for all eigenvalues $\alpha$ of $A$;

(iii) the vectors $\vec{u}, A \vec{u}, \ldots, A^{r-1} \vec{u}$ generate $\mathbb{C}^{r}$.

The proof is left for readers.

For $A \in M_{\text {reg }}$, set

$$
\xi_{i}(A)=A^{i}+\beta_{1}(A) A^{i-1}+\cdots+\beta_{i}(A) \mathbb{I}_{r} \in M_{r}(\mathbb{C}) \quad(i=1, \ldots, r-1),
$$

where $\beta_{1}(A), \ldots, \beta_{r}(A)$ are coefficients of the characteristic polynomial of $A, \operatorname{det}\left(y \mathbb{I}_{r}-A\right)=$ $y^{r}+\beta_{1}(A) y^{r-1}+\cdots+\beta_{r}(A)$.

Lemma 8. Let $A \in M_{\text {reg }}$ and $\vec{u} \in V(A)$.

(i) The matrix $g(\vec{u}, A)=\left(\vec{u}, \xi_{1}(A) \vec{u}, \ldots, \xi_{r-1}(A) \vec{u}\right) \in M_{r}(\mathbb{C})$ is invertible. Moreover, it satisfies

$$
g(\vec{u}, A)^{-1} A g(\vec{u}, A)=\left(\begin{array}{cccc}
-\beta_{1}(A) & \cdots & -\beta_{r-1}(A) & -\beta_{r}(A) \\
1 & 0 & \cdots & 0 \\
\vdots & \ddots & \ddots & \vdots \\
0 & \cdots & 1 & 0
\end{array}\right) .
$$

(ii) $g(\vec{u}, A)$ makes $B \in M_{r}(\mathbb{C})$ into the following form

$$
g(\vec{u}, A)^{-1} B g(\vec{u}, A)=\left(\begin{array}{cccc}
* & \cdots & * & * \\
* & \cdots & * & 0 \\
\vdots & \vdots & \vdots & \vdots \\
* & \cdots & * & 0
\end{array}\right)
$$

if and only if $\vec{u}$ is an eigenvector of $B \xi_{r-1}(A)$. Moreover the $(1, r)$-th entry of the RHS is equal to the eigenvalue.

Proof. (i) The invertibility of $g$ follows from Lemma 7. Another claim is checked by a direct computation.

(ii) Let $\tilde{B}=g(\vec{u}, A)^{-1} B g(\vec{u}, A)$. If $\tilde{B}$ has the form of the RHS, we obtain $B \xi_{r-1}(A) \vec{u}=\tilde{B}_{1 r} \vec{u}$ by comparing the $r$-th columns of $B g$ and $g \tilde{B}$. Conversely if $\vec{u}$ is an eigenvalue of $B \xi_{r-1}(A)$, then we see by direct calculation that $\tilde{B}_{1 r}$ is equal to its eigenvalue and $\tilde{B}_{j r}=0$ for $2 \leq j \leq r$.

Lemma 9. Let $A \in M_{\mathrm{reg}}, B \in M_{r}(\mathbb{C})$ and assume that $\operatorname{det} A=0$.

(i) $\xi_{r-1}(A)=c \vec{v}_{0}(A) \otimes{ }^{t} \vec{v}_{0}\left({ }^{t} A\right)$ for some $c \in \mathbb{C}^{\times}$.

(ii) $B \vec{v}_{0}(A)$ is an eigenvector of $B \xi_{r-1}(A)$.

Proof. (i) By the assumption on $A$, the rank of $\xi_{r-1}(A)$ is one, and $\xi_{r-1}$ satisfies $A \xi_{r-1}(A)=$ $\xi_{r-1}(A) A=0$. Thus $\xi_{r-1}(A)$ have to be written as $c \vec{v}_{0}(A) \otimes{ }^{t} \vec{v}_{0}\left({ }^{t} A\right)$ with some $c \in \mathbb{C}^{\times}$.

(ii) By (i), any $\vec{w} \in \mathbb{C}^{r}$ satisfy $\xi_{r-1}(A) \vec{w}=c \vec{v}_{0}(A)$ with some $c \in \mathbb{C}^{\times}$. By multiplying the both sides by $B$ from the left and setting $\vec{w}=B \vec{v}_{0}(A)$, we see that $B \vec{v}_{0}(A)$ is an eigenvector of $B \xi_{r-1}(A)$.

Proofs of Proposition 5. (i) We write $S(x)=S_{d} x^{d}+S_{d-1} x^{d-1}+\cdots+S_{0} \in \mathcal{S}_{\infty}$ as

$$
S(x)=\left(\begin{array}{cccc}
-\beta_{1} & \cdots & -\beta_{r-1} & 0 \\
1 & 0 & \cdots & 0 \\
\vdots & \ddots & \ddots & \vdots \\
0 & \cdots & 1 & 0
\end{array}\right) x^{d}+\left(\begin{array}{cccc}
* & \cdots & * & \beta \\
* & \cdots & * & 0 \\
\vdots & \vdots & \vdots & \vdots \\
* & \cdots & * & 0
\end{array}\right) x^{d-1}+\text { lower terms in } x
$$


where $\beta \neq 0$. Then it is easy to see $\operatorname{det} S_{d}=0$, and we can set $\vec{v}_{0}\left(S_{d}\right)={ }^{t}(0, \ldots, 0,1)$. A direct calculation shows that $\vec{u}:=S_{d-1} \vec{v}_{0}\left(S_{d}\right)={ }^{t}(\beta, 0, \ldots, 0)$ and $S_{d}$ satisfy

$$
\operatorname{det}\left(\vec{u}, S_{d} \vec{u}, \ldots, S_{d}^{r-1} \vec{u}\right)=\operatorname{det}(\operatorname{diag}(\beta, \ldots, \beta))=\beta^{r} \neq 0
$$

Thus we see $\vec{u} \in V\left(S_{d}\right)$ due to Lemma 7 , and the claim follows.

(ii) It is easy to see that $M_{\infty}$ is invariant under the action of $P G L_{r}(\mathbb{C})$, thus the map

$$
\mu: \mathcal{S}_{\infty} \times P G L_{r}(\mathbb{C}) \rightarrow M_{\infty} ;(S(x), g) \mapsto g S(x) g^{-1}
$$

is well-defined. In the following we show that $\mu$ is bijection.

First we show the surjectivity of $\mu$. For $A(x)=A_{d} x^{d}+A_{d-1} x^{d-1}+\cdots+A_{0} \in M_{\infty}$, set $g=g\left(A_{d-1} \vec{v}_{0}\left(A_{d}\right), A_{d}\right)$. By Lemma $7, g$ is invertible and $g^{-1} A_{d} g \in \Omega$ by Lemma 8(i). By Lemma 7 and $9, A_{d-1} \vec{v}_{0}\left(A_{d}\right)$ is an eigenvector of $A_{d-1} \xi_{r-1}\left(A_{d}\right)$ belonging to a nonzero eigenvalue. Thus $g^{-1} A_{d-1} g \in \mathcal{T}$ by Lemma 8(ii). Consequently we obtain $S(x)=g A(x) g^{-1} \in \mathcal{S}_{\infty}$, i.e. $\mu(S(x), g)=A(x)$.

To check the injectivity of $\mu$, we only have to check the following: for any $S(x) \in \mathcal{S}_{\infty}$, $g \in G L_{r}(\mathbb{C})$ satisfies $g^{-1} S(x) g \in \mathcal{S}_{\infty}$ only when $g$ is a scalar matrix. When $S(x)$ is given by (15), we get

$$
\begin{aligned}
& \xi_{r-1}\left(S_{d}\right)={ }^{t}(0, \ldots, 0,1) \cdot\left(1, \beta_{1}, \ldots, \beta_{r-1}\right) \\
& \left(\xi_{k}\left(S_{d}\right)\right)_{j 1}=\delta_{k, j-1} \quad \text { for } \quad k=1, \ldots, r-2
\end{aligned}
$$

The first equation implies that $\left(S_{d-1} \xi_{r-1}\left(S_{d}\right)\right)_{i j}=\delta_{i, 1} \delta_{j, 1} \beta$. This matrix has only one nonzero eigenvalue $\beta$ and the corresponding eigenvector is ${ }^{t}(a, 0, \ldots, 0)$ for some $a \in \mathbb{C}^{\times}$. By Lemma 8 (ii), we only have to show that $g\left({ }^{t}(a, 0, \ldots, 0), S_{d}\right)=c \mathbb{I}_{r}$ for some $c \in \mathbb{C}^{\times}$. This follows from the second equation.

In closing, we give some remarks. The space $M_{\infty}(14)$ is an affine subspace of

$$
\left\{A(x) \in M_{r}\left(S_{d}\right) \mid H_{r d}^{(r)}=0\right\}
$$

which is the codimension one subspace of $\mathcal{M}(r, d)$. As a generalization of Lemma 2, we easily obtain the following:

Lemma 10. If $\phi(x) \in S_{d+1}$, then (2) induces the Poisson structure on $M_{\infty} / P G L_{r}(\mathbb{C}) \simeq \mathcal{S}_{\infty}$.

The space of representatives introduced by Donagi and Markman [2, Lemma 4.1] is a subspace of $\mathcal{S}_{\infty}$ (14) defined by

$$
\left\{S(x)=S_{d} x^{d}+\cdots+S_{0} \in \mathcal{S}_{\infty} \mid \operatorname{Tr} S(x)=0, \beta_{1}\left(S_{d}\right)=\cdots=\beta_{r-1}\left(S_{d}\right)=0\right\} .
$$

The phase space discussed in $[10, \S 3.2]$ is obtained by removing the first condition in the above.

\section{A On the Poisson structure (2)}

Assume that $\phi(x)$ is a monic polynomial with only simple roots, $a_{1}, \ldots, a_{d+2}$. Consider the following isomorphism $\varphi[1,(5.6)]$ :

$$
\varphi: M_{r}\left(S_{d+1}\right) \rightarrow M_{r}(\mathbb{C})^{\oplus d+2}, A(x) \mapsto\left(c_{1} A\left(a_{1}\right), \ldots, c_{d+2} A\left(a_{d+2}\right)\right),
$$


where $c_{\alpha}=\prod_{\beta \neq \alpha}\left(a_{\alpha}-a_{\beta}\right)^{-1}$. The inverse of $\varphi^{-1}$ is given by the Lagrange interpolation formula:

$$
\varphi^{-1}: M_{r}(\mathbb{C})^{d+2} \rightarrow M_{r}\left(S_{d+1}\right), \quad\left(A^{(1)}, \ldots, A^{(d+2)}\right) \mapsto \sum_{\alpha=1}^{d+2} A^{(\alpha)} \prod_{\beta \neq \alpha}\left(x-a_{\beta}\right) .
$$

Then the pullback by $\varphi$ of the canonical Poisson structure on $M_{r}(\mathbb{C})^{\oplus d+2}$ :

$$
\left\{A_{i j}^{(\alpha)}, A_{k l}^{(\beta)}\right\}=\delta_{\alpha, \beta}\left(\delta_{j, k} A_{i l}^{(\alpha)}-\delta_{i, l} A_{k j}^{(\alpha)}\right) \quad \text { for } \quad\left(A^{(1)}, \ldots, A^{(d+2)}\right) \in M_{r}(\mathbb{C})^{\oplus d+2},
$$

is equal to (2). This is easily checked if one uses the elementary identity

$$
A(x) \phi(y)-\phi(x) A(y)=(y-x) \sum_{\alpha=1}^{d+2} A^{(\alpha)} \prod_{\mu \neq \alpha}\left(x-a_{\mu}\right)\left(y-a_{\mu}\right) .
$$

\section{B On the $G_{r}$-action (8)}

In the construction of Poisson structures in [5], the isomorphism $\varphi$ given in (16) and the following $G_{r}$-action on $M_{r}(\mathbb{C})^{\oplus d+2}$ were used:

$$
G_{r} \ni g(x):\left(A^{(1)}, \ldots, A^{(d+2)}\right) \mapsto\left(g\left(a_{\alpha}\right)^{-1} A^{(\alpha)} g\left(a_{\alpha}\right)\right)_{1 \leq \alpha \leq d+2},
$$

where $a_{\alpha} \neq a_{\beta}$ if $\alpha \neq \beta$. We show that this action is compatible with the $G_{r}$-action (8) under the isomorphism $\varphi$ when $\phi(x)=\prod_{\alpha=1}^{d+2}\left(x-a_{\alpha}\right)$.

From (17), we have

$$
\left(\varphi^{-1} \circ g(x) \circ \varphi\right) A(x)=\sum_{\alpha=1}^{d+2} c_{\alpha} g\left(a_{\alpha}\right)^{-1} A\left(a_{\alpha}\right) g\left(a_{\alpha}\right) \prod_{\mu \neq \alpha}\left(x-a_{\mu}\right) .
$$

On the other hand, substituting $x=a_{\alpha}$ into (9), we have $\tilde{A}\left(a_{\alpha}\right)=g\left(a_{\alpha}\right)^{-1} A\left(a_{\alpha}\right) g\left(a_{\alpha}\right)$. Then expressing $\tilde{A}(x)$ by the Lagrange interpolation formula, we see that $\tilde{A}(x)=\left(\varphi^{-1} \circ g(x) \circ \varphi\right) A(x)$.

\section{Acknowledgements}

The authors thank Takao Yamazaki for discussion and reading the manuscript. Y.K. is a research fellow of the Japan Society for the Promotion of Science.

\section{References}

[1] Beauville A., Jacobiennes des courbes spectrales et systèmes hamiltoniens complètement intégrables, Acta Math. 164 (1990), 211-235.

[2] Donagi R., Markman E., Spectral covers, algebraically completely integrable, Hamiltonian systems, and moduli of bundles, Lecture Notes in Math., Vol. 1620, Springer, Berlin, 1996, 1-119.

[3] Fernandes R.L., Vanhaecke P., Hyperelliptic Prym varieties and integrable systems, Comm. Math. Phys. 221 (2001), 169-196, math-ph/0011051.

[4] Fu B., Champs de vecteurs invariants par translation sur les jacobiennes affines des courbes spectrales, C. R. Math. Acad. Sci. Paris 337 (2003), no. 2, 105-110.

[5] Inoue R., Konishi Y., Yamazaki T., Jacobian variety and integrable system - after Mumford, Baeuville and Vanhaecke, J. Phys. Geom., to appear, math-ph/0512033.

[6] Mumford D., Tata lectures on theta II, Birkhäuser, 1984. 
[7] Nakayashiki A., Smirnov F.A., Cohomologies of affine Jacobi varieties and integrable systems, Comm. Math. Phys. 217 (2001), 623-652, math-ph/0001017.

[8] Pendroni M., Vanhaecke P., A Lie algebraic generalization of the Mumford system, its symmetries and its multi-Hamiltonian structure, Regul. Chaotic Dyn. 3 (1998), 132-160.

[9] Reyman A.G., Semenov-Tian-Shansky M.A., Group-theoretical methods in the theory of finite-dimensional integrable systems, Encyclopedia of Mathematical Sciences, Vol. 16, Springer-Verlag, Berlin Heidelgerg, 1994, $116-225$.

[10] Smirnov F.A., Zeitlin V., Affine Jacobi varieties of spectral curves and integrable models, math-ph/0203037.

[11] Vanhaecke P., Linearising two-dimensional integrable systems and the construction of action-angle variables, Math. Z. 211 (1992), 265-313.

[12] Vanhaecke P., Integrable systems in the realm of algebraic geometry, Lecture Notes in Math., Vol. 1638, Springer, Berlin, 2001. 